УДК 519.248:[004.8+33+301+159.9]

\title{
Postulating the Theory of Experience and Chance
}

\author{
Oleg Yu. Vorobyev* \\ Institute of Mathematics and Computer Science \\ Siberian Federal University \\ Svobodny, 79, Krasnoyarsk, 660041
}

Russia

Received 09.07.2019, received in revised form 13.08.2019, accepted 20.09.2019

The aim of the paper is the axiomatic justification of the theory of experience and chance, one of the dual halves of which is the Kolmogorov probability theory. The author's main idea was the natural inclusion of Kolmogorov's axiomatics of probability theory in a number of general concepts of the theory of experience and chance. The main result of this work is the axiom of co revent, intended for the sake of constructing a theory formed by dual theories of believabilities and probabilities, each of which itself is postulated by its own Kolmogorov system of axioms. Of course, other systems of postulating the theory of experience and chance can be imagined, however, in this work a preference is given to a system of postulates that is able to describe in the most simple manner the results of what I call an experienced-random experiment.

Keywords: eventology, event, co event, experience, chance, to experience, to happen, to occur, theory of experience and chance, theory of co events, axiom of co event, probability, believability, certainty, probability theory, believability theory, certainty theory.

DOI: 10.17516/1997-1397-2019-12-6-705-717.

\section{Co event as a set of dual pairs}

I'll start with one detail at which you should linger. Among the reasons that gave rise to the theory of experience and chance, for a long time it would be possible to linger on the philosophy of the duality of being. But our milestone is quite different.

The "tacit" Kolmogorov axiom defines each event $x$ as some subset $x \subseteq \Omega$ of elementary outcomes $\omega \in \Omega$ such that

- when a one $\omega \in x$ happens,

they say that the event $x$ happens;

- otherwise, when no elementary outcome $\omega \in x$ happens, they say that the event $x$ does not happen.

So, the fact that the event $x$ happens for an elementary outcome $\omega \in \Omega$ is defined by the "tacit" Kolmogorov axiom as a realization of the membership relation: $\omega \in x$ (see Axiom 0 on page 711). For reasons unknown to us, this postulate is not included in Kolmogorov's axiomatics of probability theory explicitly: it received from its creator the role of only a preliminary definition.

At the same time, it is this statement, as the axiom of the event, that can serve as an essential aid in delimiting probability theory and general measure theory. Moreover, in the new theory of experience and chance (TEC) [9] this axiom of the event enters as one of the dual halves in the axiom of the co being (see Axiom 1 on page 711), without explicit support for which the new

*ovorobov@sfu-kras.ru

(c) Siberian Federal University. All rights reserved 
theory can not take place because the TEC sees in everything, that we have always understood under the events, dual pairs ${ }^{\dagger}$ :

$$
\langle\text { bra-event (experience) }| \text { ket-event (chance) }\rangle \text {. }
$$

and defines its central concept, co event (experience chance), as the set of such dual pairs.

The definition of co event as a set of dual pairs (1) is not someone's whim, and certainly not mine. I will venture to say that this is only the "wish" of Kolmogorov's theory of probability, which despite its perseverance is still hidden from prying eyes. And the point is this.

It suffices to imagine a finite set of Kolmogorov events $\mathfrak{X} \subset \mathcal{A}$, chosen from the sigmaalgebra of the probability space $(\Omega, \mathcal{A}, \mathbf{P})$, which consists of Kolmogorov events $x \in \mathfrak{X}$, defined in Kolmogorov's theory of probability as measurable subsets $x \subseteq \Omega$ of elementary outcomes $\omega \in \Omega$; so that before your eyes there is such the following chain of two relations of membership:

$$
\omega \in x \in \mathfrak{X},
$$

where the Kolmogorov event $x \subseteq \Omega$ acts in dual roles: an element of the set $\mathfrak{X}$, and a subset of the set $\Omega$.

Such a dilemma is not only uncommon in a hard-to-see corpus of mathematical theories using the language of set theory, but rather it is truism. But in probability theory, this truism has proved to be a natural carrier of the deep sense of definition (1), which suggests working with each concept of the Kolmogorov theory of events and their probabilities as with a dual pair consisting of an experience of observers and an observation of chance. As a result of such element-set duality, the Cartesian product

$$
\langle\Omega \mid \Omega\rangle=\langle\Omega|\times| \Omega\rangle,
$$

of the bra-set $\langle\Omega|$ (the set of experiences of observes) and the ket-set $|\Omega\rangle$ (the set of chances of observation) becomes the mathematical model of $\Omega$, and the dual pair

$$
\langle x \mid x\rangle \subseteq\langle\Omega \mid \Omega\rangle
$$

becomes the mathematical model of each event $x \subseteq \Omega$ as the co event. The first element of the pair, the bra-event $\langle x| \subseteq\langle\Omega|$, plays a role of the event $x$ as an element of the set $\mathfrak{X}$ and describes an experience of observer of what $x$ was, and the second element, the ket-event $|x\rangle \subseteq|\Omega\rangle$, plays a dual role of the event $x$ as a subset of the set $\Omega$ and describes an observation of what $x$ is.

Moreover, a duality of an element and a subset [8], which naturally manifests itself in the concept of the Kolmogorov event in probability theory, ensures the continuation of the chain of two membership relations (2) to the following:

$$
\omega \in x \in X \in \mathfrak{Z}^{\mathfrak{X}} \subseteq \mathcal{P}(\mathfrak{X}),
$$

where now a subset of the Kolmogorov events $X \subseteq \mathfrak{X}$ also appears in the dual role as an element of the set $\mathfrak{Z}^{\mathfrak{X}} \subseteq \mathcal{P}\left(\mathfrak{X}-\left\{\varnothing^{\Omega}\right\}\right)$, and a subset of the set $\mathfrak{X}$. This time another dual pair:

$$
\left\langle\operatorname{Ter}_{X / / \mathfrak{X}} \mid \operatorname{ter}(X / / \mathfrak{X})\right\rangle \subseteq\langle\Omega \mid \Omega\rangle
$$

becomes the mathematical model of each so called terraced event numbered by $X$ as the terraced co event. The left element of the pair, terraced bra-event

$$
\left\langle\operatorname{Ter}_{X / / \mathfrak{X}}\right|=\sum_{x \in X}\langle x| \subseteq\langle\Omega|
$$

\footnotetext{
${ }^{\dagger}$ This one and a number of subsequent formulas use the bra-ket terminology and bra-ket notation $\langle\cdot \mid \cdot\rangle$, which are defined below and which largely rely on what I call an element-set labelling (see [8]).
} 
numbered by $X \subseteq \mathfrak{X}$, as by subset of the set $\mathfrak{X}$, is defined by the union of subset of experiences of observes $\langle x|, x \in X$, and the right element, terraced ket-event

$$
|\operatorname{ter}(X / / \mathfrak{X})\rangle=\bigcap_{x \in X}|x\rangle \bigcap_{x \in \mathfrak{X}-X}|x\rangle^{c} \subseteq|\Omega\rangle
$$

numbered by $X \in \mathfrak{Z}^{\mathfrak{X}}$, as by element of the set $\mathrm{Z}^{\mathfrak{X}}$, is defined as the observation of intersection of the set of chances $|x\rangle, x \in X$ and $|x\rangle^{c}=|\Omega\rangle-|x\rangle, x \in \mathfrak{X}-X$, where $|x\rangle^{c}=|\Omega\rangle-|x\rangle$ is a complement of the ket-event $|x\rangle$ to the ket-set $|\Omega\rangle$.

Although the previous preliminary text "slightly" runs ahead and contains some mathematical misunderstandings due to the premature use of the still-unknown bra-ket concepts and notations of the element-set labelling, but we will still have time and the possibility of their correct definition to show convenience, practicality and unbearable fruitfulness of dual mathematical models co event (4) and terraced co event (6) as dual pairs that are unusually effective not only in theory but also in applications.

\section{2. "Element-set coordinates" generated by a binary relation}

Our goal is to divide each concept of the theory of experience and chance into two dual parts and present it in the form of a conveniently written dual pair. For the recording of such dual pairs, we are proposing, for the time being, only formally to borrow the Dirac notation $[1,2]$, which are quite suitable for our purposes and well-proven in quantum mechanics. In order to continue the study of the duality of elements and sets in bra-ket notations, it is necessary to begin with the definition of some preliminary terminological set-theoretic constructions necessary for constructing the bra-ket presentation of the new theory. It is a question of the notion of a measurable binary relation as the most suitable applicant for the mathematical model of an event as a dual pair. It turned out that the measurable binary relation has very convenient labelling properties [8]. The point is that for work in a set-theoretic space whose objects of interest serve simultaneously space elements, sets of elements, and sets of subsets of elements, it is necessary to have in stock a certain coordinate system suitable for labelling both the space itself and its parts. Here, in my opinion, a slightly peculiar but effective system of set-theoretic coordinates, generated by the measurable binary relation and quite based on some labelling set $\mathfrak{X}$ and some set $\mathfrak{Z}^{\mathfrak{X}} \subseteq \mathcal{P}(\mathfrak{X})$ of its labelling subsets, and also on the $M$-complement $t^{\ddagger} \mathfrak{X}^{(c)}$ of the labelling set $\mathfrak{X}$ and on the one-to-one corresponding $\mathfrak{Z}^{\mathfrak{X}}$ set of its labelling subsets $\mathfrak{Z}^{\mathfrak{X}^{(c)}}=\left\{X^{c(c)}: X \in \mathfrak{Z}^{\mathfrak{X}}\right\} \subseteq \mathcal{P}\left(\mathfrak{X}^{(c)}\right)$.

Consider the measurable space $(\Omega, \mathcal{A})$ composed of some set $\Omega$ and a sigma-algebra $\mathcal{A}$ of its subsets and we emphasize that: elements $\omega \in \Omega$; measurable subsets $x \subseteq \Omega$; some set $\mathfrak{X}=\{x: x \in \mathcal{A}\} \subseteq \mathcal{A}$, composed from measurable subsets $x \in \mathfrak{X}$; and some set $\mathfrak{Z}^{\mathfrak{X}} \subseteq \mathcal{P}(\mathfrak{X}-$ $\left.\left\{\varnothing^{\Omega}\right\}\right)$ of subsets $\bar{X} \subseteq \mathfrak{X}$, consisting from measurable subsets $x \in X \subseteq \mathfrak{X}$; until they have no meaningful interpretation and form only a basis $\boldsymbol{\Lambda}$ peculiar element-set labels $\lambda \in \boldsymbol{\Lambda}$ (tags, dockets, tickets, or names), intended for a element-set labelling, or a nominating the parts and details of the construction that we are going to propose in the theory of experience and chance as a mathematical model of an event as a dual pair.

Predefinition 1 (Basic element-set labels). Basic element-set labels $\lambda \in \boldsymbol{\Lambda}$ are called as elements, sets and sets of subsets of the measurable space $(\Omega, \mathcal{A})$, and also results of terraced set-theoretic operations over them, equipped with their own titles.

\footnotetext{
${ }^{\ddagger}$ The set $\mathfrak{X}^{(c)}=\left\{x^{c}: x \in \mathfrak{X}\right\}$ is called a complement by Minkowski (an M-complement) of the set $\mathfrak{X}$.
} 
We'll fill up the stock of $\boldsymbol{\Lambda}$ tags with one more label, Cartesian product

$$
\mathfrak{X} \times \mathfrak{Z}^{\mathfrak{X}}=\left\{(x, X): x \in \mathfrak{X}, X \in \mathfrak{Z}^{\mathfrak{X}}\right\},
$$

which defines a binary relation

$$
\mathcal{R}_{\mathfrak{X}, \mathfrak{Z}^{\mathfrak{x}}}=\left\{(x, X): x \in X, x \in \mathfrak{X}, X \in \mathfrak{Z}^{\mathfrak{X}}\right\} \subseteq \mathfrak{X} \times \mathfrak{Z}^{\mathfrak{X}}
$$

as a membership relation $x \in X$ between elements $x \in \mathfrak{X}$ and subsets $X \in \mathfrak{Z}^{\mathfrak{X}}$; and also a complementary binary relation

$$
\mathcal{R}_{\mathfrak{X}, \mathfrak{Z}^{\mathfrak{x}}}^{c}=\left\{(x, X): x \notin X, x \in \mathfrak{X}, X \in \mathfrak{Z}^{\mathfrak{X}}\right\} \subseteq \mathfrak{X} \times \mathfrak{Z}^{\mathfrak{X}}
$$

as a non-membership relation $x \notin X$ between elements $x \in \mathfrak{X}$ and subsets $X \in \mathfrak{Z}^{\mathfrak{X}}$; so that

$$
\mathcal{R}_{\mathfrak{X}, \mathrm{Z}^{\mathfrak{x}}}+\mathcal{R}_{\mathfrak{X}, \mathrm{Z}^{\mathfrak{x}}}^{c}=\mathfrak{X} \times \mathfrak{Z}^{\mathfrak{X}} .
$$

Finally, we add to the stock $\boldsymbol{\Lambda}$ so called terraced ${ }^{\S}$ label

$$
\left(\operatorname{Ter}_{X / / \mathfrak{X}}, \quad \operatorname{ter}(X / / \mathfrak{X})\right)=\left(\bigcup_{x \in X} x, \bigcap_{x \in X} x \bigcap_{x \in \mathfrak{X}-X}(\Omega-x)\right) \subseteq \Omega \times \Omega,
$$

numbered by labels-subsets $X \in \mathfrak{Z}^{\mathfrak{X}}$ and while defined simply as a pair of indicated measurable subsets of $\Omega$.

To have a full stock we'll stock up in the literal sense "complementary" element-set labels, constructed from: 1) the complements $x^{c}=\Omega-x$ to measurable subsets $x \subseteq \Omega, 2$ ) the $M$ complementary set $\mathfrak{X}^{(c)}=\left\{x^{c}: x \in \mathfrak{X}\right\} \subseteq \mathcal{A}$ composed from these complements, and 3) the sets $\mathfrak{Z}^{\mathfrak{X}^{(c)}}=\left\{X^{c(c)}: X \in \mathfrak{Z}^{\mathfrak{X}}\right\} \subseteq \mathcal{P}\left(\mathfrak{X}^{(c)}\right)$ of subsets $X^{c(c)}=\left(X^{c}\right)^{(c)}=(\mathfrak{X}-X)^{(c)} \subseteq \mathfrak{X}^{(c)}$, i.e., such that $X^{c(c)}=\left\{x^{c}: x \in X^{c}\right\} \in \mathcal{Z}^{\mathfrak{X}^{(c)}}$.

There we also place a label similar to (9), the Cartesian product

$$
\mathfrak{X}^{(c)} \times \mathfrak{Z}^{\mathfrak{X}^{(c)}}=\left\{\left(x^{c}, X^{c(c)}\right): x^{c} \in \mathfrak{X}^{(c)}, X^{c(c)} \in \mathfrak{Z}^{\mathfrak{X}^{(c)}}\right\},
$$

which defines analogous to (11) a complementary binary relation

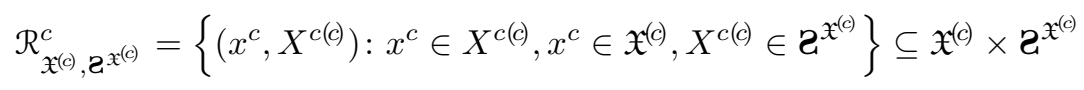

as a membership relation $x^{c} \in X^{c(c)}$ between elements $x^{c} \in \mathfrak{X}^{(c)}$ and subsets $X^{c(c)} \in \mathcal{Z}^{\mathfrak{X}^{(c)}}$; and also a complementary binary relation

$$
\mathcal{R}_{\mathfrak{X}^{(c)}, \mathfrak{Z}^{\mathfrak{X}^{(c)}}}=\left\{\left(x^{c}, X^{c(c)}\right): x^{c} \notin X^{c(c)}, x^{c} \in \mathfrak{X}^{(c)}, X^{c(c)} \in \mathfrak{Z}^{\mathfrak{X}^{(c)}}\right\} \subseteq \mathfrak{X}^{(c)} \times \mathfrak{Z}^{\mathfrak{X}^{(c)}}
$$

§Those who are familiar with the beginnings of the eventological theory $[5,2007]$ should keep their attention to the amazing inevitability of the "splitting" of the previously unified concept of the terrace event into two dual halves, the right of which is the terraced ket-event which is defined as a terrace event of the first kind $\operatorname{ter}(X / / \mathfrak{X})=\bigcap_{x \in X} x \bigcap_{x \in \mathfrak{X}-X}(\Omega-x) \subseteq \Omega$ from the eventological part of the Kolmogorov probability theory, and the left one is a terraced bra-event, a new concept from the theory of believabilities, dual to the probability theory, which is defined as terraced event of the 5th kind $\operatorname{Ter}_{X / / x}=\bigcup_{x \in X} x \subseteq \Omega$ from the eventological classification. 
as a non-membership relation $x^{c} \notin X^{c(c)}$ between elements $x^{c} \in \mathfrak{X}^{(c)}$ and subsets $X^{c(c)} \in \mathfrak{Z}^{\mathfrak{X}^{(c)}}$; so that

$$
\mathcal{R}_{\mathfrak{X}^{(c)}, \mathfrak{Z}^{\mathfrak{X}^{(c)}}}+\mathcal{R}_{\mathfrak{X}^{(c)}, \mathfrak{Z}^{\mathfrak{X}^{(c)}}}=\mathfrak{X}^{(c)} \times \mathfrak{Z}^{\mathfrak{X}^{(c)}} .
$$

Finally, do not forget the similar to (13) terrace label

$$
\left(\operatorname{Ter}_{X^{c}(c) / / \mathfrak{X}^{(c)}}, \operatorname{ter}\left(X^{c(c)} / / \mathfrak{X}^{(c)}\right)\right)=\left(\bigcup_{x^{c} \in X^{c(c)}} x^{c}, \quad \bigcap_{x^{c} \in X^{c(c)}} x^{c} \bigcap_{x^{c} \in \mathfrak{X}^{(c)}-X^{c(c)}}\left(\Omega-x^{c}\right)\right) \subseteq \Omega \times \Omega,
$$

numbered by labels-subsets $X^{c(c)} \in \mathfrak{Z}^{\mathfrak{X}^{(c)}}$.

The stock $\boldsymbol{\Lambda}$ of element-set labels $\lambda \in \boldsymbol{\Lambda}$ is intended to construct such a system of element-set "coordinates", which, relying on a duality "element-set", will allow us to divide each concept of the theory of experience and chance (TEC) into two dual parts and present it in the form of a conveniently written dual pair, i.e., pairs composed of two dual parts. In the bra-ket notation [8], the dual parts of pairs labelled with the labels $\lambda, \lambda^{\prime} \in \boldsymbol{\Lambda}$, are denoted by $\langle\lambda|$ and $\left|\lambda^{\prime}\right\rangle$ correspondingly, the entire dual pair is denoted by $\left\langle\lambda \mid \lambda^{\prime}\right\rangle$ and is defined as the Cartesian product $\left\langle\lambda \mid \lambda^{\prime}\right\rangle=\left\langle\lambda|\times| \lambda^{\prime}\right\rangle$ of their dual parts, placing the corresponding concept of the theory of experience and chance in the system of "element-set coordinates".

\section{Co event as a binary relation}

Let $\langle\Omega, \mathcal{A} \mid \Omega, \mathcal{A}\rangle=(\langle\Omega \mid \Omega\rangle,\langle\mathcal{A} \mid \mathcal{A}\rangle)$ be a measurable bra-ket space ${ }^{\mathbb{1}}$, labelled by the measurable binary relation $\mathcal{R} \subseteq\langle\Omega \mid \Omega\rangle$ using $\mathcal{R}$-labels from the measurable space $(\Omega, \mathcal{A})$ with $\mathcal{R}$-labelling sets $\mathfrak{X}_{\mathcal{R}} \subseteq \mathcal{A}$ and $\mathfrak{Z}^{\mathfrak{X}_{\mathcal{R}}} \subseteq \mathcal{P}\left(\mathfrak{X}_{\mathcal{R}}\right)$, which are defined the following way [8].

Definition 1 (basic $\mathcal{R}$-labelling set $\mathfrak{X}_{\mathcal{R}}$ ). The basic $\mathcal{R}$-labelling set $\mathfrak{X}_{\mathcal{R}} \subseteq \mathcal{A}$ of measurable subset of $\Omega$ is defined by the binary relation $\mathcal{R} \subseteq\langle\Omega \mid \Omega\rangle$ as the set of labels

$$
\mathfrak{X}_{\mathcal{R}}=\left\{x \in \mathcal{A}:|x\rangle=\left.\mathcal{R}\right|_{\left\langle\omega^{*}\right|},\left\langle\omega^{*}\right| \in\langle\Omega|\right\} \subseteq \mathcal{A}
$$

composed from measurable subsets $x \subseteq \Omega$ labelling ket-subsets $|x\rangle \subseteq|\Omega\rangle$ that serve by values of the cross-sections: $|x\rangle=\left.\mathcal{R}\right|_{\left\langle\omega^{*}\right|} \subseteq|\Omega\rangle$ of binary relation $\mathcal{R}$ by bra-points $\left\langle\omega^{*}\right| \in\langle\Omega|$.

Note, if there is the bra-point $\left\langle\omega^{*}\right| \in\langle\Omega|$ such that $\left.\mathcal{R}\right|_{\left\langle\omega^{*}\right|}=\varnothing_{|\Omega\rangle}$ then $\left.\mathcal{R}\right|_{\left\langle\omega^{*}\right|}=\left|\varnothing^{\Omega}\right\rangle$, i.e. the empty cross-section $\left.\mathcal{R}\right|_{\left\langle\omega^{*}\right|}$ coincides with the ket-subset $\left|\varnothing^{\Omega}\right\rangle$ where $\varnothing^{\Omega} \in \mathfrak{X}_{\mathcal{R}}$ is the $\Omega$-empty label.

Definition 2 (basic set $\mathrm{Z}^{\mathfrak{X}_{\mathcal{R}}}$ of $\mathcal{R}$-labelling subsets). The basic set $己^{\mathfrak{X}_{\mathcal{R}}} \subseteq$ $\mathcal{P}\left(\mathfrak{X}_{\mathcal{R}}-\left\{\varnothing^{\Omega}\right\}\right)$ of $\mathcal{R}$-labelling subsets of measurable subsets of $\Omega$ is defined by the binary relation $\mathcal{R} \subseteq\langle\Omega \mid \Omega\rangle$ as the set of set-labels

$$
\mathfrak{Z}^{\mathfrak{X}_{\mathcal{R}}}=\left\{X \subseteq \mathfrak{X}_{\mathcal{R}}-\left\{\varnothing^{\Omega}\right\}: \operatorname{ter}\left(X / / \mathfrak{X}_{\mathcal{R}}\right) \neq \varnothing^{\Omega}\right\} \subseteq \mathcal{P}\left(\mathfrak{X}_{\mathcal{R}}-\left\{\varnothing^{\Omega}\right\}\right),
$$

composed only from labelling subsets $X \subseteq \mathfrak{X}_{\mathcal{R}}$ that do not contain the $\Omega$-empty label: $\varnothing^{\Omega} \notin X$, and number the $\Omega$-nonempty terraced labels: $\operatorname{ter}\left(X / / \mathfrak{X}_{\mathcal{R}}\right) \neq \varnothing^{\Omega}$.

The measurable relation $\mathcal{R}$ generates the following element-set $\mathcal{R}$-labelling quotient-sets.

$$
\langle\Omega| / \mathcal{R}=\left\langle\mathfrak{X}_{\mathcal{R}}\right|=\left\{\langle x|: x \in \mathfrak{X}_{\mathcal{R}}\right\}
$$

\footnotetext{
IIn the following discourse, I use the notions and notations of the element-set labelling, introduced in the preliminary work $[8,9]$, without necessarily defining them again here for the sake of space saving.
} 
is the $\mathcal{R}$-labelling bra-quotient-set $\langle\Omega| / \mathcal{R}$ by the binary relation $\mathcal{R} \subseteq\langle\Omega \mid \Omega\rangle$, under which the labels $x \in \mathfrak{X}_{\mathcal{R}}$ of labelling set $\mathfrak{X}_{\mathcal{R}}$ label all bra-subsets $\langle x| \in\langle\Omega| / \mathcal{R}$ of the quotient-set $\langle\Omega| / \mathcal{R}$;

$$
|\Omega\rangle / \mathcal{R}=\left|\mathfrak{Z}^{\mathfrak{X}_{\mathcal{R}}}\right\rangle=\left\{\left|\operatorname{ter}\left(X / / \mathfrak{X}_{\mathcal{R}}\right)\right\rangle: X \in \mathfrak{Z}^{\mathfrak{X}_{\mathcal{R}}}\right\}
$$

is the $\mathcal{R}$-labelling ket-quotient-set $|\Omega\rangle / \mathcal{R}$ by the binary relation $\mathcal{R} \subseteq\langle\Omega \mid \Omega\rangle$, under which the subsets $X \in \mathcal{Z}^{\mathfrak{X}_{\mathcal{R}}}$ from the set of labelling subsets $\boldsymbol{Z}^{\mathfrak{X}_{\mathcal{R}}}$ label the terraced ket-subsets $\left|\operatorname{ter}\left(X / / \mathfrak{X}_{\mathcal{R}}\right)\right\rangle \in|\Omega\rangle / \mathcal{R}$ of the quotient-set $|\Omega\rangle / \mathcal{R}$;

$$
\langle\Omega \mid \Omega\rangle / \mathcal{R}=\left\langle\mathfrak{X}_{\mathcal{R}} \mid \mathfrak{Z}^{\mathfrak{X}_{\mathcal{R}}}\right\rangle=\left\{\left\langle x \mid \operatorname{ter}\left(X / / \mathfrak{X}_{\mathcal{R}}\right)\right\rangle: x \in \mathfrak{X}_{\mathcal{R}}, X \in \mathfrak{Z}^{\mathfrak{X}_{\mathcal{R}}}\right\}
$$

is the $\mathcal{R}$-labelling bra-ket-quotient-set $\langle\Omega \mid \Omega\rangle / \mathcal{R}$ by the binary relation $\mathcal{R} \subseteq\langle\Omega \mid \Omega\rangle$, under which the pairs $(x, X)$, where $x \in \mathfrak{X}_{\mathcal{R}}$ is an element of the labelling set $\mathfrak{X}_{\mathcal{R}}$, and $X \in \mathfrak{Z}^{\mathfrak{X}_{\mathcal{R}}}$ is a subset from the set $\mathfrak{Z}^{\mathfrak{X}_{\mathcal{R}}}$ of labelling subsets, label all bra-ket-subsets $\left\langle x \mid \operatorname{ter}\left(X / / \mathfrak{X}_{\mathcal{R}}\right)\right\rangle \in\langle\Omega \mid \Omega\rangle / \mathcal{R}$ of the quotient-set $\langle\Omega \mid \Omega\rangle / \mathcal{R}$.

Predefinition 2 (events and co events).

$\star$ The bra-points $\langle\omega|\in| \Omega\rangle$ are called elementary bra-incomes (incomes).

$\star$ The bra-subsets $\langle x| \subseteq\langle\Omega|$ and terraced bra-subsets $\left\langle\operatorname{Ter}_{X / / \mathfrak{X}_{\mathcal{R}}}\right| \subseteq\langle\Omega|$ of the bra-set $\langle\Omega|$ are called bra-events and terraced bra-events correspondingly.

* The ket-points $|\omega\rangle \in|\Omega\rangle$ are called elementary ket-outcomes (outcomes).

$\star$ The ket-subsets $|x\rangle \subseteq|\Omega\rangle$ and terraced ket-subsets $\left|\operatorname{ter}\left(X / / \mathfrak{X}_{\mathcal{R}}\right)\right\rangle \subseteq|\Omega\rangle$ of the ket-set $|\Omega\rangle$ are called ket-events and terraced ket-events correspondingly.

$\star$ The bra-ket-subsets $\langle x \mid x\rangle \subseteq\langle\Omega \mid \Omega\rangle,\left\langle\operatorname{Ter}_{X / / \mathfrak{X}_{\mathcal{R}}} \mid \operatorname{ter}\left(X / / \mathfrak{X}_{\mathcal{R}}\right)\right\rangle \subseteq\langle\Omega \mid \Omega\rangle$ and $\left\langle x \mid \operatorname{ter}\left(X / / \mathfrak{X}_{\mathcal{R}}\right)\right\rangle \subseteq$ $\langle\Omega \mid \Omega\rangle$ are called elementary bra-ket-events.

$\star$ The bra-ket-subset $\mathcal{R} \subseteq\langle\Omega \mid \Omega\rangle$, i.e., any measurable binary relation, generating the $\mathcal{R}$ labelling, is called a co event (an experienced-random co $\sim$ event).

Predefinition 3 (full-believable, certainty, non-experienced, and impossible events and full-believable-certainty and non-experienced-impossible co events).

$\star$ The bra-events $\langle\Omega|$ and $\langle\varnothing|$ are called full-believable and non-experienced correspondingly.

$\star$ The ket-events $|\Omega\rangle$ and $|\varnothing\rangle$ are called certainty and impossible correspondingly.

$\star$ The co events $\langle\Omega \mid \Omega\rangle$ and $\langle\varnothing \mid \varnothing\rangle$ are called full-believable-certainty and non-experiencedimpossible correspondingly.

$\star$ The co events $\langle\Omega \mid x\rangle$ and $\langle x \mid \Omega\rangle$ are called full-believable-random and experienced-certainty correspondingly.

* The co events $\langle\varnothing \mid x\rangle$ and $\langle x \mid \varnothing\rangle$ are called non-experienced-random and experiencedimpossible correspondingly.

$\star$ The co events $\langle\Omega \mid \varnothing\rangle$ and $\langle\varnothing \mid \Omega\rangle$ are called full-believable-impossible and non-experiencedcertainty correspondingly.

Predefinition 4 (R-labelled events). For the sake of brevity, the following gen- 
eral notation of $\mathcal{R}$-labelled events, and suited general denotations:

$$
\begin{aligned}
& \left\langle\lambda_{\mathcal{R}}^{*}\right|= \begin{cases}\langle x|, & x \in \mathfrak{X}_{\mathcal{R}}, \\
\left\langle\operatorname{Ter}_{X / / \mathfrak{X}_{\mathcal{R}}}\right|, & X \in \mathfrak{Z}^{\mathfrak{X}_{\mathcal{R}}},\end{cases} \\
& \left|\lambda_{\mathcal{R}}\right\rangle= \begin{cases}|x\rangle, & x \in \mathfrak{X}_{\mathcal{R}}, \\
\left|\operatorname{ter}\left(X / / \mathfrak{X}_{\mathcal{R}}\right)\right\rangle, & X \in \mathfrak{Z}^{\mathfrak{X}_{\mathcal{R}}},\end{cases} \\
& \left\langle\lambda_{\mathcal{R}}^{*} \mid \lambda_{\mathcal{R}}\right\rangle= \begin{cases}\langle x \mid x\rangle, & x \in \mathfrak{X}_{\mathcal{R}}, \\
\left\langle\operatorname{Ter}_{X / / \mathfrak{X}_{\mathcal{R}}} \mid \operatorname{ter}\left(X / / \mathfrak{X}_{\mathcal{R}}\right)\right\rangle, & X \in \mathfrak{C}^{\mathfrak{X}_{\mathcal{R}}},\end{cases} \\
& \left\langle\lambda_{\mathcal{R}}^{*} \mid \lambda_{\mathcal{R}}^{\prime}\right\rangle=\left\langle x \mid \operatorname{ter}\left(X / / \mathfrak{X}_{\mathcal{R}}\right)\right\rangle, x \in \mathfrak{X}_{\mathcal{R}}, X \in \mathfrak{Z}^{\mathfrak{X}_{\mathcal{R}}}
\end{aligned}
$$

are introduced for ket-events $|x\rangle \subseteq|\Omega\rangle$, terraced ket-events $\left|\operatorname{ter}\left(X / / \mathfrak{X}_{\mathcal{R}}\right)\right\rangle \subseteq|\Omega\rangle$, bra-events $\langle x| \subseteq$


$\left\langle\operatorname{Ter}_{X / / \mathfrak{X}_{\mathcal{R}}} \mid \operatorname{ter}\left(X / / \mathfrak{X}_{\mathcal{R}}\right)\right\rangle \subseteq\langle\Omega \mid \Omega\rangle$ and $\left\langle x \mid \operatorname{ter}\left(X / / \mathfrak{X}_{\mathcal{R}}\right)\right\rangle \subseteq\langle\Omega \mid \Omega\rangle$; which are defined in Predefinition 3 and labelled by the co event $\mathcal{R} \subseteq\langle\Omega \mid \Omega\rangle$.

Predefinition 5 (bra-ket-duality of $\mathcal{R}$-labelled events). They say that the $\mathcal{R}$-labelled bra-event $\left\langle\lambda_{\mathcal{R}}^{*}\right|$ and the $\mathcal{R}$-labelled ket-event $\left|\lambda_{\mathcal{R}}\right\rangle$ are bra-ket-dual each other and form the pair of bra-ket-dual events as the Cartesian product $\left\langle\lambda_{\mathcal{R}}^{*} \mid \lambda_{\mathcal{R}}\right\rangle=\left\langle\lambda_{\mathcal{R}}^{*}|\times| \lambda_{\mathcal{R}}\right\rangle$.

\section{4. "Something happens when that is experienced, what happens"}

\subsection{The axiom of co event as of what occurs, when that is experienced, what happens}

Before the axioms 1 (the axiom of co event), which is central to the theory of experience and chance, I will formulate for comparison, in the same notation, what I called the "silent" Kolmogorov axiom. Its number is zero.

Axiom 0 (an event happens, when its elementary outcome happens [Kolmogorov theory of probabilities]).

(1) The elementary outcome $\omega \in \Omega$ is what happens: $\omega=\omega^{\uparrow}$, or does not happen: $\omega \neq \omega^{\uparrow}$.

(2) Any event $\lambda \subseteq \Omega$ happens: $\lambda=\lambda^{\uparrow}$, when the elementary outcome happens: $\omega=\omega^{\uparrow}$, which belong to it: $\omega^{\uparrow} \in \lambda$.

Axiom 1 (co event occurs, when that is experienced, what happens [theory of experience and chance]).

(1) The elementary ket-outcome $|\omega\rangle \in|\Omega\rangle$ is what happens: $|\omega\rangle=|\omega\rangle^{\uparrow}$, or does not happen: $|\omega\rangle \neq|\omega\rangle^{\uparrow}$.

(2) For any $\mathcal{R} \subseteq\langle\Omega \mid \Omega\rangle$ any $\mathcal{R}$-labelled ket-event $\left|\lambda_{\mathcal{R}}\right\rangle \subseteq|\Omega\rangle$ happens: $\left|\lambda_{\mathcal{R}}\right\rangle=\left|\lambda_{\mathcal{R}}\right\rangle^{\uparrow}$, when the elementary outcome happens: $|\omega\rangle=|\omega\rangle^{\uparrow}$, which belong to it: $|\omega\rangle^{\uparrow} \in\left|\lambda_{\mathcal{R}}\right\rangle$. 
(3) For any $\mathcal{R} \subseteq\langle\Omega \mid \Omega\rangle$ any $\mathcal{R}$-labelled bra-event $\left\langle\lambda_{\mathcal{R}}^{*}\right| \subseteq\langle\Omega|$ is experienced: $\left\langle\lambda_{\mathcal{R}}^{*}\right|=\left\langle\left.\lambda_{\mathcal{R}}^{*}\right|^{\downarrow}\right.$, when dual $\mathcal{R}$-labelled ket-event happens: $\left|\lambda_{\mathcal{R}}\right\rangle=\left|\lambda_{\mathcal{R}}\right\rangle^{\uparrow}$.

(4) The elementary bra-income $\left\langle\omega^{*}\right| \in\langle\Omega|$ is expereinced $\left\langle\omega^{*}\right|=\left\langle\left.\omega^{*}\right|^{\downarrow}\right.$, when $\mathcal{R}$-labelled bra-event: $\left\langle\lambda_{\mathcal{R}}^{*}\right|=\left\langle\left.\lambda_{\mathcal{R}}^{*}\right|^{\downarrow}\right.$ is experienced, to which $\left\langle\omega^{*}\right|$ belongs: $\left\langle\omega^{*}\right| \in\left\langle\left.\lambda_{\mathcal{R}}^{*}\right|^{\downarrow}\right.$.

(5) The elementary income-outcome $\left\langle\omega^{*} \mid \omega\right\rangle \in\langle\Omega \mid \Omega\rangle$ is what occurs: $\left\langle\omega^{*} \mid \omega\right\rangle=\left\langle\omega^{*} \mid \omega\right\rangle^{\downarrow \uparrow}$, when the elementary ket-outcome: $|\omega\rangle=|\omega\rangle^{\uparrow}$ happens and the elementary bra-income: $\left\langle\omega^{*}\right|=\left\langle\left.\omega^{*}\right|^{\downarrow}\right.$ is experiencred; or does not occur: $\left\langle\omega^{*} \mid \omega\right\rangle \neq\left\langle\omega^{*} \mid \omega\right\rangle^{\downarrow \uparrow}$, when $|\omega\rangle \neq|\omega\rangle^{\uparrow}$ or $\left\langle\omega^{*}\right| \neq\left\langle\left.\omega^{*}\right|^{\downarrow}\right.$.

(6) The co event $\mathcal{R} \subseteq\langle\Omega \mid \Omega\rangle$ occurs: $\mathcal{R}=\mathcal{R}^{\downarrow \uparrow}$, when the elementary income-outcome: $\left\langle\omega^{*} \mid \omega\right\rangle=\left\langle\omega^{*} \mid \omega\right\rangle^{\downarrow \uparrow}$ occurs, which belongs to it: $\left\langle\omega^{*} \mid \omega\right\rangle^{\downarrow \uparrow} \in \mathcal{R}$.

\subsection{Kolmogorov axioms}

\section{Kolmogorov axioms of believability theory}

Let $\langle\Omega|$ be the bra-set of bra-points $\langle\omega| \in\langle\Omega|$, which we shall call the elementary bra-incomes (or simply the elementary incomes), and $\langle\mathcal{A}|$ be the set of subsets from $\langle\Omega|$. For any $\mathcal{R} \subseteq\langle\Omega \mid \Omega\rangle$ elements $\left\langle\lambda_{\mathcal{R}}^{*}\right| \in\langle\mathcal{A}|$ are called the $\mathcal{R}$-labelled bra-events, and $\langle\Omega|$ be the bra-set of elementary incomes.

Axiom 2 (algebra of bra-events). $\quad\langle\mathcal{A}|$ is an algebra of bra-events. The algebra of bra-events is also called the bra-algebra.

Axiom 3 (believability of bra-events). For any $\mathcal{R} \subseteq\langle\Omega \mid \Omega\rangle$ each $\mathcal{R}$-labelled braevent $\left\langle\lambda_{\mathcal{R}}^{*}\right| \in\langle\mathcal{A}|$ is assigned the nonnegative real number $\mathbf{B}\left(\left\langle\lambda_{\mathcal{R}}^{*}\right|\right)$. This number is called the believability of $\mathcal{R}$-labelled bra-event $\left\langle\lambda_{\mathcal{R}}^{*}\right|$.

Axiom 4 (normalization of believability). $\mathbf{B}(\langle\Omega|)=1$.

Axiom 5 (additivity of believability). If $\mathcal{R}$-labelled bra-events $\left\langle\lambda_{\mathcal{R}}^{*}\right|$ and $\left\langle\lambda_{\mathcal{R}}^{* \prime}\right|$ are not intersected in $\langle\Omega|$, then

$$
\mathbf{B}\left(\left\langle\lambda_{\mathcal{R}}^{*}\right|+\left\langle\lambda_{\mathcal{R}}^{* \prime}\right|\right)=\mathbf{B}\left(\left\langle\lambda_{\mathcal{R}}^{*}\right|\right)+\mathbf{B}\left(\left\langle\lambda_{\mathcal{R}}^{* \prime}\right|\right) .
$$

Axiom 6 (continuity of believability). For a decreasing sequence $\left\langle\left.\lambda_{\mathcal{R}}^{*}\right|_{1} \supseteq\left\langle\left.\lambda_{\mathcal{R}}^{*}\right|_{2} \supseteq\right.\right.$ $\ldots \supseteq\left\langle\left.\lambda_{\mathcal{R}}^{*}\right|_{n} \supseteq \ldots\right.$ of $\mathcal{R}$-labelled bra-events from $\langle\mathcal{A}|$ such that $\bigcap_{n}\left\langle\left.\lambda_{\mathcal{R}}^{*}\right|_{n}=\varnothing^{\langle\Omega|}\right.$, the equality $\lim _{n} \mathbf{B}\left(\left\langle\left.\lambda_{\mathcal{R}}^{*}\right|_{n}\right)=0\right.$ takes place.

Aggregate of objects $\langle\Omega, \mathcal{A}, \mathbf{B}|=(\langle\Omega|,\langle\mathcal{A}|, \mathbf{B})$, which is satisfied to axioms $2,3,4,5$ and 6 we shall call the believability bra-space, or simply the believability space.

\section{Kolmogorov axioms of probability theory}

Let $|\Omega\rangle$ be the ket-set of ket-points $|\omega\rangle \in|\Omega\rangle$ which we shall call the elementary ket-outcomes (or simply the elementary outcomes), and $|\mathcal{A}\rangle$ be the set of subsets from $|\Omega\rangle$. For any $\mathcal{R} \subseteq\langle\Omega \mid \Omega\rangle$ the elements $\left|\lambda_{\mathcal{R}}\right\rangle \in|\mathcal{A}\rangle$ of the set $|\mathcal{A}\rangle$ we shall call the $\mathcal{R}$-labelled ket-events, and $|\Omega\rangle$ be the ket-set of elementary outcomes.

Axiom 7 (algebra of ket-events). ket-events is also called the ket-algebra.
$|\mathcal{A}\rangle$ is an algebra of ket-events. The algebra of 
Axiom 8 (probability of ket-events). For any $\mathcal{R} \subseteq\langle\Omega \mid \Omega\rangle$ each $\mathcal{R}$-labelled ketevent $\left|\lambda_{\mathcal{R}}\right\rangle \in|\mathcal{A}\rangle$ is assigned the nonnegative real number $\mathbf{P}\left(\left|\lambda_{\mathcal{R}}\right\rangle\right)$. This number is called the probability of $\mathcal{R}$-labelled ket-event $\left|\lambda_{\mathcal{R}}\right\rangle$.

Axiom 9 (normalization of probability). $\mathbf{P}(|\Omega\rangle)=1$.

Axiom 10 (additivity of probability). If $\mathcal{R}$-labelled ket-events $\left|\lambda_{\mathcal{R}}\right\rangle$ and $\left|\lambda_{\mathcal{R}}^{\prime}\right\rangle$ are not intersected in $|\Omega\rangle$, then

$$
\mathbf{P}\left(\left|\lambda_{\mathcal{R}}\right\rangle+\left|\lambda_{\mathcal{R}}^{\prime}\right\rangle\right)=\mathbf{P}\left(\left|\lambda_{\mathcal{R}}\right\rangle\right)+\mathbf{P}\left(\left|\lambda_{\mathcal{R}}^{\prime}\right\rangle\right)
$$

Axiom 11 (continuity of probability). For a decreasing sequence $\left|\lambda_{\mathcal{R}}\right\rangle_{1} \supseteq\left|\lambda_{\mathcal{R}}\right\rangle_{2} \supseteq$ $\ldots \supseteq\left|\lambda_{\mathcal{R}}\right\rangle_{n} \supseteq \ldots$ of $\mathcal{R}$-labelled ket-events from $|\mathcal{A}\rangle$ such that $\bigcap_{n}\left|\lambda_{\mathcal{R}}\right\rangle_{n}=\varnothing^{|\Omega\rangle}$ the equality $\lim _{n} \mathbf{P}\left(\left|\lambda_{\mathcal{R}}\right\rangle_{n}\right)=0$ takes place.

The aggregate of oblects $|\Omega, \mathcal{A}, \mathbf{P}\rangle=(|\Omega\rangle,|\mathcal{A}\rangle, \mathbf{P})$, which is satisfied to axioms 7, 8, 9, 10 and 11 we shall call the probability ket-space, or simply the probability space.

\subsection{Axioms of the theory of certainties (believabilities-probabilities)}

Let $\langle\Omega \mid \Omega\rangle=\langle\Omega|\times| \Omega\rangle$ be the set of bra-ket-points $\left\langle\omega^{*} \mid \omega\right\rangle=\left\langle\omega^{*}|\times| \omega\right\rangle \in\langle\Omega \mid \Omega\rangle$, which we shall call the elementary bra-ket-incomes-outcomes (or simply the elementary incomes-outcomes), and $\langle\mathcal{A} \mid \mathcal{A}\rangle$ be the set of subsets from $\langle\Omega \mid \Omega\rangle$. For any $\mathcal{R} \subseteq\langle\Omega \mid \Omega\rangle$ the elements $\left\langle\lambda_{\mathcal{R}}^{*} \mid \lambda_{\mathcal{R}}\right\rangle \in\langle\mathcal{A} \mid \mathcal{A}\rangle$ are called the $\mathcal{R}$-labelled bra-ket-events, and $\langle\Omega \mid \Omega\rangle$ be the bra-ket-set of elementary incomes-outcomes.

Axiom 12 (algebra of bra-ket-events). $\quad\langle\mathcal{A} \mid \mathcal{A}\rangle=\alpha(\langle\mathcal{A}|\times| \mathcal{A}\rangle)$ is a minimal algebra of bra-ket-events, which contains the Cartesian product of algebras $\langle\mathcal{A}|\times| \mathcal{A}\rangle$. This algebra is also called bra-ket-algebra.

Axiom 13 (certainty of bra-ket-events). For any $\mathcal{R} \subseteq\langle\Omega \mid \Omega\rangle$ each $\mathcal{R}$-labelled bra-ketevent $\left\langle\lambda_{\mathcal{R}}^{*} \mid \lambda_{\mathcal{R}}\right\rangle \in\langle\mathcal{A} \mid \mathcal{A}\rangle$ is assigned the nonnegative real number $\mathbf{\Phi}\left(\left\langle\lambda_{\mathcal{R}}^{*} \mid \lambda_{\mathcal{R}}\right\rangle\right)=\mathbf{B}\left(\left\langle\lambda_{\mathcal{R}}^{*}\right|\right) \mathbf{P}\left(\left|\lambda_{\mathcal{R}}\right\rangle\right)$. This number is called the certainty of $\mathcal{R}$-labelled bra-ket-event $\left\langle\lambda_{\mathcal{R}}^{*} \mid \lambda_{\mathcal{R}}\right\rangle$.

Property 1 (normalization of certainty). $\boldsymbol{\Phi}(\langle\Omega \mid \Omega\rangle)=1$.

Proof. $\boldsymbol{\Phi}(\langle\Omega \mid \Omega\rangle)=\mathbf{B}(\langle\Omega|) \mathbf{P}(|\Omega\rangle)=1$ by axioms 4,9 and 13 .

Property 2 (additivity of certainty). If $\mathcal{R}$-labelled bra-ket-events $\left\langle\lambda_{\mathcal{R}}^{*} \mid \lambda_{\mathcal{R}}\right\rangle$ and $\left\langle\lambda_{\mathcal{R}}^{* \prime} \mid \lambda_{\mathcal{R}}^{\prime}\right\rangle$ are not intersected in $\langle\Omega \mid \Omega\rangle$, then

$$
\boldsymbol{\Phi}\left(\left\langle\lambda_{\mathcal{R}}^{*} \mid \lambda_{\mathcal{R}}\right\rangle+\left\langle\lambda_{\mathcal{R}}^{* \prime} \mid \lambda_{\mathcal{R}}^{\prime}\right\rangle\right)=\boldsymbol{\Phi}\left(\left\langle\lambda_{\mathcal{R}}^{*} \mid \lambda_{\mathcal{R}}\right\rangle\right)+\boldsymbol{\Phi}\left(\left\langle\lambda_{\mathcal{R}}^{* \prime} \mid \lambda_{\mathcal{R}}^{\prime}\right\rangle\right)
$$

Proof. An additivity of product of additive functions $\mathbf{B}$ and $\mathbf{P}$ is a fact of general measure theory. So that an additivity of certainty $\boldsymbol{\Phi}$ on $\langle\mathcal{A} \mid \mathcal{A}\rangle$ follows routinely from the axioms 5,10 and 13 .

Property 3 (continuity of certainty). For a decreasing sequence $\left\langle\lambda_{\mathcal{R}}^{*} \mid \lambda_{\mathcal{R}}\right\rangle_{1} \supseteq\left\langle\lambda_{\mathcal{R}}^{*} \mid \lambda_{\mathcal{R}}\right\rangle_{2} \supseteq$ $\ldots \supseteq\left\langle\lambda_{\mathcal{R}}^{*} \mid \lambda_{\mathcal{R}}\right\rangle_{n} \supseteq \ldots$ of $\mathcal{R}$-labelled bra-ket-events from $\langle\mathcal{A} \mid \mathcal{A}\rangle$ such that $\bigcap_{n}\left\langle\lambda_{\mathcal{R}}^{*} \mid \lambda_{\mathcal{R}}\right\rangle_{n}=\varnothing^{\langle\Omega \mid \Omega\rangle}$, the equality $\lim _{n} \boldsymbol{\Phi}\left(\left\langle\lambda_{\mathcal{R}}^{*} \mid \lambda_{\mathcal{R}}\right\rangle_{n}\right)=0$ takes place. 
Proof. A continuity of certainty $\Phi$ on $\langle\mathcal{A} \mid \mathcal{A}\rangle$ as a product of the continuous believability $\mathbf{B}$ on $\langle\mathcal{A}|$ and the continuous probability $\mathbf{P}$ on $|\mathcal{A}\rangle$ follows from the general measure theory by axioms 6,11 and 13 .

The aggregate of objects $\langle\Omega, \mathcal{A}, \mathbf{B} \mid \Omega, \mathcal{A}, \mathbf{P}\rangle=(\langle\Omega \mid \Omega\rangle,\langle\mathcal{A} \mid \mathcal{A}\rangle, \mathbf{\Phi})$, which is satisfied to axioms 12 and 13 we shall call the certainty (believability-probability) bra-ket-space, or simply the certainty space.

Property 4 (believability, probability and certainty of some events and corevent). From the axioms of the theory of certainties it follows that

$$
\begin{aligned}
& \star \mathbf{\Phi}(\langle\Omega \mid \Omega\rangle)=\mathbf{B}(\langle\Omega|)=\mathbf{P}(|\Omega\rangle)=1, \\
& \star \mathbf{\Phi}(\langle\varnothing \mid \varnothing\rangle)=\mathbf{B}(\langle\varnothing|)=\mathbf{P}(|\varnothing\rangle)=0, \\
& \star \mathbf{\Phi}(\langle\Omega \mid \varnothing\rangle)=\mathbf{\Phi}(\langle\varnothing \mid \Omega\rangle)=0, \\
& \star \mathbf{\Phi}\left(\left\langle\varnothing \mid \lambda_{\mathcal{R}}\right\rangle\right)=\mathbf{\Phi}\left(\left\langle\lambda_{\mathcal{R}}^{*} \mid \varnothing\right\rangle\right)=0, \\
& \star \mathbf{\Phi}\left(\left\langle\Omega \mid \lambda_{\mathcal{R}}\right\rangle\right)=\mathbf{P}\left(\left|\lambda_{\mathcal{R}}\right\rangle\right), \\
& \star \mathbf{\Phi}\left(\left\langle\lambda_{\mathcal{R}}^{*} \mid \Omega\right\rangle\right)=\mathbf{B}\left(\left\langle\lambda_{\mathcal{R}}^{*}\right|\right) .
\end{aligned}
$$

\subsection{Properties of co $\sim$ events and its dual halves: bra-events and ket-events}

Property 5 (bra-event is experienced, when ket-event happens). If the ket-event $|x\rangle \subseteq|\Omega\rangle$ happens: $|x\rangle=|x\rangle^{\uparrow}$, then the bra-event $\langle x| \subseteq \Omega$ is experienced: $\langle x|=\left\langle\left. x\right|^{\downarrow}\right.$. Otherwise, when ket-event $|x\rangle \subseteq|\Omega\rangle$ does not happen: $|x\rangle \neq|x\rangle^{\uparrow}$, the bra-event isn't experienced: $\langle x| \neq\left\langle\left. x\right|^{\downarrow}\right.$.

Proof follows from the item (3) of Axiom 1.

Property 6 (bra-events from which something follows; ket-events that follow from something).

(1) If the ket-event $|x\rangle \subseteq|\Omega\rangle$ happens: $|x\rangle=|x\rangle^{\uparrow}$, then all ket-events which contain it: $|x\rangle^{\uparrow} \subseteq|y\rangle \subseteq|\Omega\rangle$ happens: $|y\rangle=|y\rangle^{\uparrow}$; in other words, all ket-events, which follow from $|x\rangle^{\uparrow}$, happens.

(2) If the bra-event $\langle x| \subseteq\langle\Omega|$ is experienced: $\langle x|=\left\langle\left. x\right|^{\downarrow}\right.$, then all bra-events, which are contained in it: $\langle y| \subseteq\left\langle\left. x\right|^{\downarrow} \subseteq\langle\Omega|\right.$, are experienced: $\langle y|=\left\langle\left. y\right|^{\downarrow}\right.$; in other words, all bra events, from which $\left\langle\left. x\right|^{\downarrow}\right.$ follows, are experienced.

Proof follows from the items (2) and (4) of Axiom 1.

Property 7 (terraced bra-event is experienced, terraced ket-event happens).

(1) The terraced ket-event

$$
\left|\operatorname{ter}\left(X / / \mathfrak{X}_{\mathcal{R}}\right)\right\rangle=\bigcap_{x \in X}|x\rangle \bigcap_{x \in \mathfrak{X}_{\mathcal{R}}-X}(|\Omega\rangle-|x\rangle) \in|\mathcal{A}\rangle
$$

happens: $\left|\operatorname{ter}\left(X / / \mathfrak{X}_{\mathcal{R}}\right)\right\rangle=\left|\operatorname{ter}\left(X / / \mathfrak{X}_{\mathcal{R}}\right)\right\rangle^{\uparrow}$, when the ket-outcome, which belongs to it: $|\omega\rangle \in$ $\left|\operatorname{ter}\left(X / / \mathfrak{X}_{\mathcal{R}}\right)\right\rangle$, happens: $|\omega\rangle=|\omega\rangle^{\uparrow}$. Otherwise, the terraced ket-event does not happens: $\left|\operatorname{ter}\left(X / / \mathfrak{X}_{\mathcal{R}}\right)\right\rangle \neq\left|\operatorname{ter}\left(X / / \mathfrak{X}_{\mathcal{R}}\right)\right\rangle^{\uparrow}$. 
(2) The terraced bra-event

$$
\left\langle\operatorname{Ter}_{X / / \mathfrak{X}_{\mathcal{R}}}\right|=\sum_{x \in X}\langle x| \in\langle\mathcal{A}|
$$

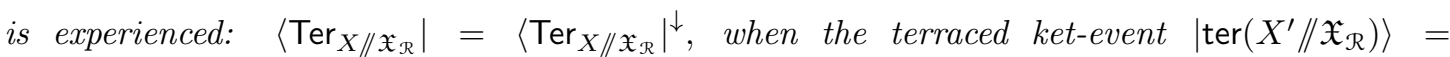
$\left|\operatorname{ter}\left(X^{\prime} / / \mathfrak{X}_{\mathcal{R}}\right)\right\rangle^{\uparrow}$, such that $X \subseteq X^{\prime}$ (see Footnotell) happens. Otherwise, the terraced bra-event



Proof of (1) follows from the item (2) of Axiom 1, and the proof of (2) follows from the item (3) of Axiom 1 and the item (1) of Property 6.

Property 8 (co event as a membership relation). Any co event $\mathcal{R} \subseteq\langle\Omega \mid \Omega\rangle$ in the measurable bra-ket-space $\langle\Omega, \mathcal{A} \mid \Omega, \mathcal{A}\rangle$ is equivalence to the membership relation

$$
\mathcal{R}_{\left\langle\mathfrak{X}_{\mathcal{R}}\right| \mathfrak{Z}^{\left.\mathfrak{X}_{\mathcal{R}}\right\rangle}}=\left\{\left\langle x \mid \operatorname{ter}\left(X / / \mathfrak{X}_{\mathcal{R}}\right)\right\rangle: x \in X\right\} \subseteq\left\langle\mathfrak{X}_{\mathcal{R}} \mid \mathfrak{Z}^{\mathfrak{X}_{\mathcal{R}}}\right\rangle
$$

on element-set $\mathcal{R}$-labelling $\left\langle\mathfrak{X}_{\mathcal{R}} \mid \mathfrak{Z}^{\mathfrak{X}_{\mathcal{R}}}\right\rangle$ of the quotient-set $\langle\Omega \mid \Omega\rangle / \mathcal{R}$. In other words,

$$
\mathcal{R}=\left\{\left\langle\omega^{*} \mid \omega\right\rangle \in\langle\Omega \mid \Omega\rangle:\left\langle\omega^{*} \mid \omega\right\rangle \in\left\langle x \mid \operatorname{ter}\left(X / / \mathfrak{X}_{\mathcal{R}}\right)\right\rangle \in \mathcal{R}_{\left\langle\mathfrak{X}_{\mathcal{R}} \mid \mathfrak{Z}^{\mathfrak{x}_{\mathcal{R}}}\right\rangle}\right\} \subseteq\langle\Omega \mid \Omega\rangle .
$$

Wherein the conevent $\mathcal{R}$ occurs then and only then, when the elementary income-outcome $\left\langle\omega^{*} \mid \omega\right\rangle=\left\langle\omega^{*} \mid \omega\right\rangle^{\downarrow \uparrow}$ occurs, such that $\left\langle\omega^{*} \mid \omega\right\rangle^{\downarrow \uparrow} \in\left\langle x \mid \operatorname{ter}\left(X / / \mathfrak{X}_{\mathcal{R}}\right)\right\rangle$, and the membership relation: $x \in X$ holds.

Proof relies on equivalence of the inclusion relation $\subseteq\langle\Omega \mid \Omega\rangle$ and the membership relation $\in_{\mathfrak{X}_{\mathcal{R}}}$ $($ see $[8,9])$

$$
\left\langle x \mid \operatorname{ter}\left(X / / \mathfrak{X}_{\mathcal{R}}\right)\right\rangle \subseteq \mathcal{R} \quad \Longleftrightarrow \quad x \in X
$$

from which it follows that the co event $\mathcal{R}$ occurs, i.e., $\left\langle\omega^{*} \mid \omega\right\rangle^{\downarrow \uparrow} \in \mathcal{R}$, then and only then, when two membership relations $\left\langle\omega^{*} \mid \omega\right\rangle^{\downarrow \uparrow} \in\left\langle x \mid \operatorname{ter}\left(X / / \mathfrak{X}_{\mathcal{R}}\right)\right\rangle$ and $x \in X$ hold. This proves the property.

\section{Instead of discussing}

Before the finish I have to slow down on three sharp corners.

On the first one, we need to stop and carefully study the main innovation of this work, Axiom 1 on page 711, which expands the "silent" Kolmogorov axiom of an event, so that this axiom together with its dual reflection allowed a new theory to jointly explore both the future randomness of observations, and the past experience of observers.

On the second, it is impossible to rush past the very curious temporal bra-ket-duality of statements from the property 6 on the page 714 , which states that

- from the Kolmogorov theory of probabilities: If there is some ket-event, then with it all ket-events occur, in which it is contained as a ket-subset. In other words, all the ket-events which follow from it, i.e. which can serve as its consequences in the future.

- from the dual theory of believabilities: If some bra-event is experienced then with it are experienced all the bra-events, which it contains as bra-subsets. In other words, all braevents from which it follows, i.e., which could serve as its causes in the past.

\footnotetext{
"In the evenology [5] this event has a special denotation: $\operatorname{ter}_{X / / \mathfrak{X}_{\mathcal{R}}}=\sum_{X \subseteq X^{\prime}} \operatorname{ter}\left(X^{\prime} / / \mathfrak{X}_{\mathcal{R}}\right)$ and is called the terraced event of the 2-d type.
} 
This remarkable property of temporal duality ket-events and bra-events clearly shows the similarity and difference between the future chance and the past experience, which for the first time are jointly mathematically correctly studied in the theory experience and chance postulated in this article (see also [10]).

And finally, on the third one, it is worthwhile once again to linger on explaining the new theory (see [9]) for quite a long time confusing the apologists of fuzzy mathematics [3,4,11-13] on the non-additivity of the set functions of interest, the origins of which are considered in my works earlier $[6,7]$.

\section{References}

[1] P.A.M.Dirac, A new notation for quantum mechanics, Proceedings of the Cambridge Philosophical Society, (1939), no. 35, 416-418

[2] P.A.M.Dirac, The principles of quantum mechanics, Yeshiva University, New York, 1964.

[3] D.Dubois, H. Prade, Possibility theory, probability theory and multiple-valued logics: A clarification, Annals of Mathematics and Artificial Intelligence, 32(2001), 35-66.

[4] G.Shafer, A mathematical theory of evidence, Princeton University Press, Princeton, NJ, 1976 .

[5] O.Yu.Vorobyev, Eventology, Siberian Federal University Press, Krasnoyarsk, Russia. Academia:38605343, Academia:189393, 2007.

[6] O.Yu.Vorobyev, Contemporary uncertainty theories: An eventological view, In. Proc. of the VIII Intern. FAM Conf. on Financial and Actuarial Mathematics and Related Fields, Krasnoyarsk, SFU, 1:2009 , 26-34.

[7] O.Yu.Vorobyev, Eventology versus contemporary theories of uncertainty, In. Proc. of the XII Intern. EM conference on eventological mathematics and related fields, Krasnoyarsk: SFU, 2009, 13-27, MPRA:13961.

[8] O.Yu.Vorobyev, An element-set-labelling a Cartesian product by measurable binary relations which leads to postulates of the theory of experience and chance as a theory of co events, In. Proc. of the XV Intern. FAMEMS Conf. on Financial and Actuarial Mathematics and Eventology of Multivariate Statistics \& the Workshop on Hilbert's Sixth Problem; Krasnoyarsk, SFU, 2016, 9-24, Academia:34390291

[9] O.Yu.Vorobyev, Postulating the theory of experience and chance as a theory of co events (co beings), In. Proc. of the XV Intern. FAMEMS Conf. on Financial and Actuarial Mathematics and Eventology of Multivariate Statistics \& the Workshop on Hilbert's Sixth Problem; Krasnoyarsk, SFU, 2016, 25-43, arXiv:1801.07147.

[10] O.Yu.Vorobyev, Theory of dual co event-based means, In. Proc. of the XV Intern. FAMEMS Conf. on Financial and Actuarial Mathematics and Eventology of Multivariate Statistics \& the Workshop on Hilbert's Sixth Problem; Krasnoyarsk, SFU, 2016), 44-93, Academia:34357251.

[11] L.A.Zadeh, Fuzzy sets, Information and Control, 8(1965), no. 3, 338-353.

[12] L.A.Zadeh, A note on similarity-based definitions of possibility and probability, Information Sciences, 267(2014), 334-336. 
[13] H.-J.Zimmermann, Fuzzy set theory and its applications, Kluwer Academic Publishers, Dordrecht, Netherlands, 2001.

\section{Постулирование теории опыта и случая}

Олег Ю. Воробьев

Institute of Mathematics and Computer Science

Siberian Federal University

Svobodny, 79, Krasnoyarsk, 660041

Russia Россия

Целъю работы является аксиоматическое обоснование теории опыта и случая, одной из дуалъных половин которой служит колмогоровская теория вероятностей. Ведущей мыслъю автора было при этом естественное включение колмогоровской аксиоматики теории вероятностей в ряд общих понятий теории опыта и случая. Главный резулътат этой работы - аксиома со бътти, предназначенная для построения теории, образованной дуальными друг другу теориями уверенностей и вероятностей, каждая из которых сама по себе постулируется собственной колмогоровской системой аксиом. Разумеется, можно представить себе и другие системы постулирования теории опьта и случая, однако в этой работе предпочтение отдается системе постулатов, которая способна наиболее простым образом описать результаты того, что называют опытнослучайным экспериментом.

Ключевые слова: эвентология, собътие, со бытие, опът, случай, испьтыватъ, случатъся, происходить, теория опыта и случая, теория со бытий, аксиома со бытия, вероятность, уверенность, определенность, теория вероятностей, теория уверенностей, теория определенностей. 